\title{
AN APPRAISAL OF THE EPIDEMIOLOGY OF TRYPANOSOMA CRUZI SEROLOGY IN BRAZIL
}

\author{
M. F. FEITOSA \& H. KRIEGER
}

Instituto Oswaldo Cruz, Departamento de Genética, Caixa Postal 926, 20001 Rio de Janeiro, RJ, Brasil

A large bibliographic survey provided data on Trypanosoma cruzi serology covering the period 1948-1984. Epidemiological-demographic methods provided an estimate of 11\% for the prevalence of positive serology in Brazil, by 1984. Significant temporal trends were observed for most of the Brazilian geographical regions as well as for Brazil, as a whole. The parabolic curve that fit best for the entire country, indicates that by 1991, the incidence of new positive serology would be close to zero. This conclusion needs further fine-adjustment, since the forecast point is somewhat distant from the measured period.

Key words: Trypanosoma cruzi serology - temporal variation - seroepidemiology

In the last decades, the Trypanosoma cruzi serology has kept the attention of many investigators, both in blood banks and in serologic field research that covered most of the Brazilian geographical regions. Although some further knowledge of the sero-epidemiologic data of $T$. cruzi infection is necessary for the understanding of the natural history of Chagas' disease as well as to disclose some patterns of the dynamics of the infection in Brazil, some broad considerations about the distribution of Chagas' disease in Brazil are already known (cf. Castro Filho \& Silveira, 1979).

Several large sero-epidemiological surveys were conducted in Brazil (Castro Filho \& Silveira, 1979; Camargo et al., 1984), providing a good picture of $T$. cruzi infection in Brazil, at a certain momment of the Chagas' disease natural history in the country.

These informations together with the available sero-epidemiological data collected by many studies, performed in different times and places could provide some further clues on the trends of this infection in Brazil. The present study is an attempt to disclose some of these trends, by means of epidemiological and demographic methodologies.

Research supported by Conselho Nacional de Desenvolvimento Científico e Tecnológico (CNPq) and Financiadora de Projetos (FINEP).

Received 15 May 1990.

Accepted 10 April 1991.

\section{MATERIALS AND METHODS}

A large bibliographical search provided data, from 1948-1984, on the serology of $T$. cruzi infection. This search included 131 articles with 196 samples, derived mainly from blood bank data and field seroepidemyological surveys. The data on prevalence of positive serology to $T$. cruzi, geographical location, sample size, as well as the source and year of publication, are . fully presented in the Appendix.

It should be stressed that the data utilized in the present study refers only to serological reaction (both complement fixation and immunofluorescence techniques). Doubtful reations were neglected.

Statistical analyses was based mainly on stepwise multiple regression, employing both the SPSS software and programs from the GENIOC library from the Department of Genetics, Oswaldo Cruz Institute (Cabello \& Krieger, 1990).

\section{RESULTS AND DISCUSSION}

The average prevalences for positive reactions against $T$. cruzi, as well as their standard deviations for each of the Brazilian geographical regions, are presented in Table I.

These values, weighted by their respective population sizes (source: Brazilian Institute of Geography and Statistics - IBGE, 1984) provided a rough estimate for the prevalence of $T$. cruzi infection in Brazil of $11.17 \%$. 
TABLE I

Distribution of positive serology against Trypanosoma cruzi

\begin{tabular}{lrr}
\hline $\begin{array}{l}\text { Geographical } \\
\text { region }\end{array}$ & Prevalence & $+/-$ SD \\
\hline North & & \\
Northeast & 0.96 & 0.91 \\
Southeast & 14.41 & 9.33 \\
South & 9.95 & 14.08 \\
Middle-West & 13.02 & 9.12 \\
\hline
\end{tabular}

TABLE II

Analyses of variance results of the most parcimonious regression models of serologic prevalence of

Trypanosoma cruzi infection on year

\begin{tabular}{llll}
\hline & D. F. F & Model \\
\hline Region & Regression Residual & \\
\hline
\end{tabular}

\begin{tabular}{|c|c|c|c|c|c|c|}
\hline \multirow{2}{*}{ North } & Prevalence & 1 & 6 & 0.024 & $\mathrm{n} . \mathrm{s}$ & no effect \\
\hline & $\begin{array}{l}\text { Ln of } \\
\text { Prevalence }\end{array}$ & 1 & 6 & 0.051 & n.s & no effes \\
\hline \multirow{2}{*}{ Northeast } & Prevalence & 2 & 91 & 16.548 & $a$ & parabol \\
\hline & $\begin{array}{l}\mathrm{LN} \text { of } \\
\text { Prevalence }\end{array}$ & 2 & 91 & 31.908 & $a$ & parabol \\
\hline \multirow{2}{*}{ Southeast } & Prevalence & 2 & 5 & 5.872 & $a$ & parabo \\
\hline & $\begin{array}{l}\text { Ln of } \\
\text { Prevalence }\end{array}$ & 1 & 56 & 2.401 & n.s & no effe \\
\hline \multirow{2}{*}{ South } & Prevalence & 1 & 19 & 5.373 & $a$ & lineas \\
\hline & $\begin{array}{l}\text { Ln of } \\
\text { Prevalence }\end{array}$ & 1 & 19 & 3.435 & $\mathrm{n} s$ & no $\mathrm{e}$ \\
\hline \multirow{2}{*}{$\begin{array}{l}\text { Middle- } \\
\text { West }\end{array}$} & Prevalence & 2 & 11 & 0.615 & n.s & no eff \\
\hline & $\begin{array}{l}\text { Ln of } \\
\text { Prevalence }\end{array}$ & 2 & 11 & 0.062 & n.s & no effe \\
\hline \multirow{2}{*}{ Brazil } & Prevalence & 2 & 192 & 17.426 & $a$ & parabolic \\
\hline & $\begin{array}{l}\text { Ln of } \\
\text { Prevalence }\end{array}$ & 2 & 192 & 20.352 & $a$ & parabo \\
\hline
\end{tabular}

a: significant at the $0.01 \mathrm{P}$ level.

Since the data cover a rather large period of time, it was tried to uncover possible temporal trends on the prevalence of positive serology, through multiple regression analyses. The results of these analyses are summarized in Table II, and ploted in Figure. As can be seen, there is no temporal effect at the Northern and Midle-West geographical regions (it should be pointed out that for the Northeastern region trere is only information on two years, cf. Appendix), while for both the Northeastern and the Southeastern regions, the temporal effect seems to be parabolic, with maximum/ minimum point at around 1963 and 1969 , respectively. As for the southern region, it seems that the temporal effect is linear and negative. The whole picture for Brazil shows a parabolic trend, similar to that of the Northeastern region.

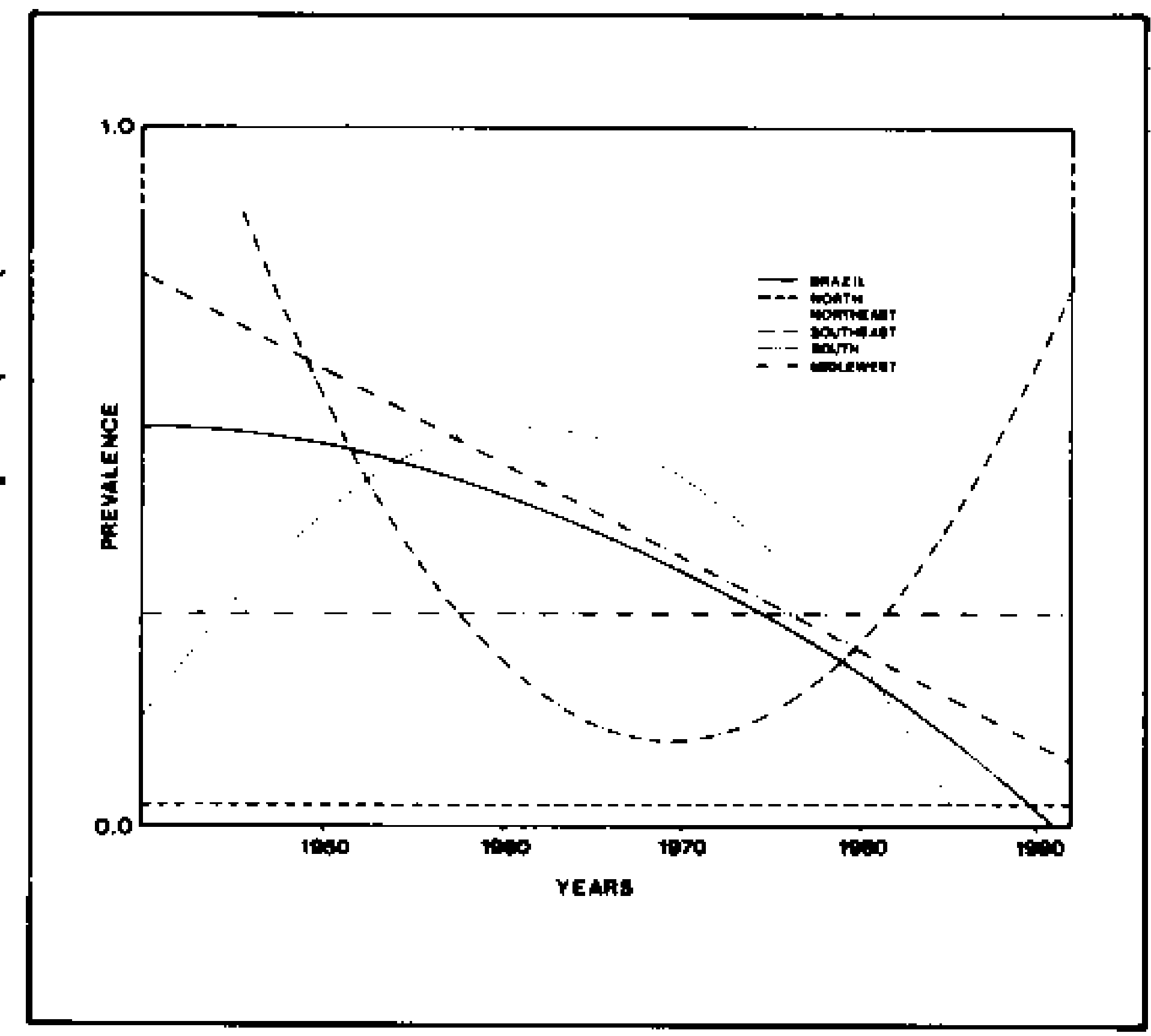

Regression plots of serological prevalence for Trypanosoma cruzi in Brazil as a whole, and subdivided by geographical regions.

In order to avoid spurious effects due to normal distribution's departure, a natural logarithm transformation was applyed to the prevalence data. These analyses were also shown in Table II. Except for the Southeastern and Southern region, the results of the previous analyses were consistent with the analyses employing the logarithm of positive serologic prevalence as dependent variable. It should be pointed out, that for the Southern region, although the $\log$ analysis did not reach the choosed level of significance (0.05), the face values of the regression coefficients are consistent with each other.

However, caution should be taken in the interpretation of this graph. As the prevalence values are assumed to be independent, the curves indicate that by around 1991 there 
would be no new cases of positive serology for Chagas" disease. Of course, this conclusion is weakly supported by statistical inference, since it is a relative distant forecast point from the employed range of observations and there is some apparent discrepancies among the region trends. Moreover, there is no support for taking as representative the prevalence figures presented by most of the reports. Nevertheless, there is no available evidence that contradicts the representativeness of this data set. A reanalysis of this problem, based on 1985-1990 data is being conducted in order to improve the prediction curves. Temporal trends of prevalence are important tools for the epidemiologist in order to uncover agents and/or nosogenic mechanisms introduced or removed from populations and to determine their effects upon the development of certain diseases. As for infections disease, it can provide valuable informations of the dynamics of transmission and on the effectiveness of adopted prophylactic measures. The techniques employed above could give some of the answers asked by public health experts and also could be applyed to other endemic diseases data in order to disclose these trends.

\section{APPENDIX}

Prevalence of Trypanosoma cruzi infection in several Brazilian locaties

\begin{tabular}{|c|c|c|c|c|c|}
\hline State & Localities & Year & $\begin{array}{c}\text { Sample } \\
\text { size }\end{array}$ & $\begin{array}{c}\% \\
\text { Positivity }\end{array}$ & References \\
\hline \multicolumn{6}{|l|}{ NORTH } \\
\hline Ronđônia & & 1984 & & 0.41 & $\begin{array}{l}\text { Camargo et al., 1984. Rev. Inst. Med. Trop., } \\
26: 192-204 \text {. }\end{array}$ \\
\hline Acre & & 1984 & & 2.39 & $\begin{array}{l}\text { Camargo et al., 1984. Rev. Inst. Med. Trop., } \\
26: 192-204 .\end{array}$ \\
\hline Amazonas & & $\begin{array}{l}1979 \\
1984\end{array}$ & 10,054 & $\begin{array}{l}1.8 \\
1.88\end{array}$ & $\begin{array}{l}\text { Castro Filho \& Silveira, 1979. Rev. Bras. } \\
\text { Malar. D. Trop., } 31: 85-98 \text {. } \\
\text { Camargo et al., 1984. Rev. Inst. Med. Trop., } \\
\text { 26: 192-204. }\end{array}$ \\
\hline Roraima & & 1984 & & 0.31 & $\begin{array}{l}\text { Camargo et al., 1984. Rev. Inst. Med. Trop., } \\
\text { 26: 192-204. }\end{array}$ \\
\hline Pará & & $\begin{array}{l}1979 \\
1984\end{array}$ & & $\begin{array}{l}0.3 \\
0.56\end{array}$ & $\begin{array}{l}\text { Castro Filho \& Silveira, 1979. Rev. Bras. } \\
\text { Malar. D. Trop., 31:85-98. } \\
\text { Camargo et al., 1984. Rev. Inst. Med. Trop., } \\
\text { 26:192-204. }\end{array}$ \\
\hline Amapá & & 1984 & & 0.0 & $\begin{array}{l}\text { Camargo et al., 1984. Rev. Inst. Med. Trop., } \\
26: 192-204 .\end{array}$ \\
\hline
\end{tabular}

\section{NORTHEAST}

Maranhão

Piauí

Oeiras

Oeiras

Ceará

(Região Cariri)

Barbalha
Castro Filho \& Silveira,

0.12 Camargo et al., 1984. Rev. Inst. Med. Trop., 26: 192-204.

12.1 Lima et al., 1977. Com XIII Congr. Soc. Bras. Med. Trop., Brasília.

3.7 Castro Filho \& Silveira, 1979. Rev. Bras. Malar. D. Trop., 31: 85-98.

4.04 Camargo et al., 1984. Rev. Inst. Med. Trop., 26: 192-204.

11132.0
Alencar et al., 1963. J. Bras. Med., 7: 593605.

Alencar et al., 1963. Rev. Bras. Malar. D. Chagas, 15: 551-565. 


\begin{tabular}{|c|c|c|c|c|c|}
\hline State & Localities & Year & $\begin{array}{l}\text { Sample } \\
\text { size }\end{array}$ & $\begin{array}{c}\% \\
\text { Positivity }\end{array}$ & References \\
\hline & Crato & 1963 & 250 & 41.0 & Ibid. \\
\hline & Jardim & 1963 & 3 & 0.0 & Ibid. \\
\hline & $\begin{array}{l}\text { Juazeiro } \\
\text { (Regiāo Baturité) }\end{array}$ & 1963 & 6 & 25.0 & Ibid. \\
\hline & Palmácia & 1963 & 53 & 15.0 & Ibid. \\
\hline & Pacoti & 1963 & 147 & 11.0 & lbid. \\
\hline & Fortaleza & 1967 & 267 & 5.2 & $\begin{array}{l}\text { Lima et al., 1967. Rev. Fac. Med. Univ. } \\
\text { Fed., Ceará,7:3-13. }\end{array}$ \\
\hline & Fortaleza & 1967 & 420 & 8.6 & Ibid. \\
\hline & & 1977 & 7.757 & 2.9 & $\begin{array}{l}\text { Alencar et al., 1977. Com. XHI Congr. Soc. } \\
\text { Bras. Med. Trop. Brasília. }\end{array}$ \\
\hline & & 1979 & 29,973 & 1.3 & $\begin{array}{l}\text { Castro Filho \& Silveira, 1979. Rev. Bras. } \\
\text { Malar. D. Trop., 31:85-98. }\end{array}$ \\
\hline & & 1984 & & 0.84 & $\begin{array}{l}\text { Camargo et al., 1984. Rev. Inst. Med. Trop., } \\
\text { 26:192-204. }\end{array}$ \\
\hline
\end{tabular}

Rio Grande do Norte

(Agreste)

Santa Cruz

(Centro Norte)

Açú

(Chapada do Apori)

Augusto Severo

Mossoró

Apodi

(Seridó)

Caiacó

Florânia

Jardim do Seridó

Acari

1970

$199 \quad 12.1$

1970

1970

1970

1970

1970

1970

1970

1970

1979

1984

1957

(Litoral-Brejo)
Bananeiras

Serraria

Pirpirituba

Santa Rosa

Espírito Santo

Campina Grande

(Caatinga

Curimataú-Cariri)

Pilar

Itabaiana

Pedra de Fogo

Ingá

Guarabira

Caiçara

Monteiro

Sumé

Taperoá

(Sertão)

Teixeira

Curema

Piancó

Patos

Patos
1957

1957

1957

1957

1957

1957

1957

1957

1957

1957

1957

1957

1957

1957

1957.

1957

1957

1957

1970
$333 \quad 27.6$

$\begin{array}{ll}169 & 23.1\end{array}$

$128 \quad 23.4$

$\begin{array}{ll}16 & 12.5\end{array}$

$50 \quad 22.0$

39910.0

$\begin{array}{ll}131 & 27.5\end{array}$

38124.1

$\begin{array}{ll}65 & 24.6\end{array}$

$\begin{array}{ll}72 & 12.5\end{array}$

$307 \quad 16.0$

$84 \quad 13.1$

$124 \quad 8.9$

628

$306 \quad 16.0$

$47 \quad 21.3$

$246 \quad 28.9$

$464 \quad 25.0$

$400 \quad 27.2$
$214 \quad 14.0$
Lucena, 1970. Rev. Bras. Malar. D. Chagas, 22: 3-173.

Ibid.

Ibid.

Ibid.

Ibid.

Ibid.

Ibid.

Ibid.

Ibid.

Castro Filho \& Silveira, 1979. Rev. Bras. Malar. D. Trop., 31: 85-98.

78 Camargo et al., 1984. Rev. Inst. Med. Thop., 26: 192-204.

Lucena \& Costa. 1957. Rev. Bras. Med., 14: 323-327.

Ibid.

Ibid.

Ibid.

Ibid.

Ibid.

lbid.

Ibid.

Ibid.

Ibid.

lbid.

Ibid.

Ibid.

Ibid.

Ibid.

Ibid.

Ibid.

Ibid.

Ibid.

Lucena, 1970. Rev. Bras. Malar. O Chagas, 22: 3-173. 


\begin{tabular}{|c|c|c|c|c|c|}
\hline State & Localities & Year & $\begin{array}{c}\text { Sample } \\
\text { size }\end{array}$ & $\begin{array}{c}\% \\
\text { Positivity }\end{array}$ & References \\
\hline & & 1979 & 42,484 & 2.3 & $\begin{array}{l}\text { Castro Filho \& Silveira, 1979. Rev. Bras. } \\
\text { Malar. D. Trop., 31:85-98. }\end{array}$ \\
\hline & & 1984 & & 3.48 & $\begin{array}{l}\text { Camargo et al., 1984. Rev. Inst. Med. Trop., } \\
\text { 26: 192-204. }\end{array}$ \\
\hline \multirow[t]{24}{*}{ Pernambuco } & Nazaré da Mata & 1954 & 410 & 20.87 & $\begin{array}{l}\text { Borba et al., 1954. Arq. Bras. Cardiol., 7: } \\
\text { 191-200. }\end{array}$ \\
\hline & Recife & 1954 & 62 & 4.84 & Ibid. \\
\hline & Timbaúba & 1955 & 1,062 & 13.43 & Marques, 1955. (cit. Lucena, 1959). \\
\hline & Recife & 1956 & 222 & 3.6 & $\begin{array}{l}\text { Silva et al., 1956. Publ. Méd., S. Paulo, 27: } \\
\text { 23-25. }\end{array}$ \\
\hline & (Litoral-Mata) & & & & \\
\hline & Aliança & 1959 & 299 & 17.1 & $\begin{array}{l}\text { Lucena, 1959. Rev. Bras. Med., 15: 864- } \\
866 .\end{array}$ \\
\hline & Catende & 1959 & 325 & 12.3 & Ibid. \\
\hline & Gemeleira & 1959 & 67 & 11.9 & Ibid. \\
\hline & Palmares & 1959 & 309 & 6.5 & lbid. \\
\hline & Nazaré da Mata & 1970 & 948 & 21.0 & $\begin{array}{l}\text { Lucena, 1970. Rev. Bras. Malar. D. Chagas, } \\
22: 3-173 .\end{array}$ \\
\hline & Timbaúba & 1970 & 631 & 20.0 & lbid. \\
\hline & $\begin{array}{l}\text { Carpina } \\
\text { (Agreste) }\end{array}$ & 1970 & 625 & 20.5 & Ibid. \\
\hline & Limoeira & 1959 & 592 & 13.3 & Ibid. \\
\hline & Bom Jardim & 1959 & 303 & 16.8 & Ibid. \\
\hline & Orobó & 1959 & 321 & 17.1 & Ibid. \\
\hline & $\begin{array}{l}\text { Surubim } \\
\text { (Sertão) }\end{array}$ & 1959 & 328 & 14.9 & Ibid. \\
\hline & Salgueiro & 1959 & 206 & 14.1 & $\begin{array}{l}\text { Lucena, 1959. Rev. Bras. Med., 15: 864- } \\
866 .\end{array}$ \\
\hline & Ouricuri & 1959 & 203 & 15.3 & Ibid. \\
\hline & Sertania & 1970 & 310 & 20.0 & $\begin{array}{l}\text { Lucena, 1970. Rev. Bras. Malar. D. Chagas, } \\
22: 3-173 \text {. }\end{array}$ \\
\hline & Pedra & 1970 & 323 & 13.6 & lbid. \\
\hline & Petrolina & 1970 & 365 & 21.1 & Ibid. \\
\hline & Recife & 1970 & 136 & 4.41 & $\begin{array}{l}\text { Huggins et al., 1970. Rev. Soc. Bras. Med. } \\
\text { Trop., 4: 105-112. }\end{array}$ \\
\hline & & 1979 & 53,754 & 2.1 & $\begin{array}{l}\text { Castro Filho \& Silveira, 1979. Rev. Bras. } \\
\text { Malar. D. Trop., 31:85-98. }\end{array}$ \\
\hline & & 1984 & & 2.79 & $\begin{array}{l}\text { Camargo et al., 1984. Rev. Inst. Med. Trop., } \\
\text { 26:192-204. }\end{array}$ \\
\hline \multirow[t]{13}{*}{ Alagoas } & (Marítma) & & & & \\
\hline & S. Luiz Quitunde & 1961 & 301 & 18.3 & $\begin{array}{l}\text { Lucena, 1961. Rev. Bras. Med., 18: 258- } \\
261 .\end{array}$ \\
\hline & $\begin{array}{l}\text { Passo Camaragibe } \\
\text { (Mata) }\end{array}$ & 1961 & 303 & 26.4 & Ibid. \\
\hline & Muruci & 1961 & 400 & 16.2 & Ibid. \\
\hline & São José da Lage & 1961 & 407 & 12.5 & Ibid. \\
\hline & Uniào dos Palmares & 1970 & 398 & 32.7 & $\begin{array}{l}\text { Lucena, 1970. Rev. Bras. Malar. D. Chagas, } \\
22: 3-173 .\end{array}$ \\
\hline & $\begin{array}{l}\text { Porto Calvo } \\
\text { (Sertāo) }\end{array}$ & 1970 & 299 & 20.7 & Ibid. \\
\hline & Santana do Ipanema & 1970 & 202 & 13.4 & Ibid. \\
\hline & Arapiraca & 1970 & 201 & 21.4 & Ibid. \\
\hline & $\begin{array}{l}\text { Palmeira dos Indios } \\
\text { (Sanfranciscana) }\end{array}$ & 1970 & 302 & 15.6 & Ibid. \\
\hline & Pão de Açúcar & 1970 & 398 & 32.7 & lbid. \\
\hline & & 1979 & 10,193 & 5.1 & $\begin{array}{l}\text { Castro Filho \& Silveira, 1979. Rev. Bras. } \\
\text { Malar. D. Trop., 31:85-98. }\end{array}$ \\
\hline & & 1984 & & 2.48 & $\begin{array}{l}\text { Camargo et al., 1984. Rev. Inst. Med. Trop., } \\
\text { 26: 192-204. }\end{array}$ \\
\hline Sergipe & & 1979 & 20,777 & 4.8 & $\begin{array}{l}\text { Castro Filho \& Silveira, 1979. Rev. Bras. } \\
\text { Malar. D. Trop., 31:85-98. }\end{array}$ \\
\hline
\end{tabular}




\begin{tabular}{lccc}
\hline State & Localities & $\begin{array}{c}\text { Sample } \\
\text { size }\end{array}$ & $\begin{array}{c}\% \\
\text { Positivity }\end{array}$ \\
\hline
\end{tabular}

\begin{tabular}{|c|c|c|c|c|c|}
\hline & & 1984 & & 5.97 & $\begin{array}{l}\text { Camargo et al., 1984. Rev. Inst. Med. Trop., } \\
26: 192-204 .\end{array}$ \\
\hline \multirow[t]{5}{*}{ Bahia } & $\begin{array}{l}\text { Salvador } \\
\text { Paulo Afonso }\end{array}$ & $\begin{array}{l}1960 \\
1968\end{array}$ & $\begin{array}{r}8,411 \\
689\end{array}$ & $\begin{array}{l}25.0 \\
2.75\end{array}$ & $\begin{array}{l}\text { Pondé, 1960. Hospital., 58: 1073-1097. } \\
\text { Salgado \& Pellegrino, 1968. (cit. Barretto, } \\
\text { 1979). }\end{array}$ \\
\hline & Castro Alves & 1976 & 1,051 & 38,3 & $\begin{array}{l}\text { Mott et al., 1976. Am J. Trop. Med., Hyg., } \\
25: 552-562 \text {. }\end{array}$ \\
\hline & $\begin{array}{l}\text { Castro Alves } \\
\text { (Fazenda Sapé) }\end{array}$ & 1979 & 166 & 33.7 & $\begin{array}{l}\text { Hoff et al., 1979. Am Soc. Trop. Med. } \\
\text { Hye. 28.461-466. }\end{array}$ \\
\hline & & 1979 & 58,969 & 7.4 & $\begin{array}{l}\text { Castro Filho \& Silveira, 1979. Rev. Bras. } \\
\text { Malar. D. Trop., } 31: 85-98 \text {. }\end{array}$ \\
\hline & & 1984 & 58,969 & 7.4 & $\begin{array}{l}\text { Camargo et al., 1984. Rev. Inst. Med. Trop., } \\
26: 192-204 .\end{array}$ \\
\hline
\end{tabular}

\section{SOUTHEAST}

Minas Gerais

Espírito Santo

Bambuí

Belo Horizonte

Belo Horizonte

(Regiāo Oeste)

Pedra Branca-

Sertãozinho

Araguari

Belo Horizonte

Uberaba

Belo Horizonte

Pains

Pains

Iguatama

Iguatama

Virgem da Lapa

Botumirim

Montalvânia

1969

6,583

0.4

1975

3,000

0.066

1979

1980

$19,193 \quad 0.2$

$4,108 \quad 0.36$

1984
Dias et al., 1948. Brasil - Médio, 62 (49, $50,51,52): 412-413$.

Pellegrino, 1949. Rev. Bras. Med., 6: 297301.

Pellegrino et al., 1951. Mem. Inst. Oswaldo Cruz, 49: 555-564.

Laranja et al., 1951. Hospital, 40: 945-988.

Dias \& Brant, 1952. An. IX Congr. Brasil. Hig., P. Alegre, p. 267-270.

Biancalana et al., 1953. Hospital, 44: 745 749.

Pellegrino, 1959. Rev. Bras. Malar. D. Trop., 11:697-706.

Jatene \& Jacomo, 1959. Rev. Goiana Med., 5: 23-30.

Salgado Pellegrino, 1963. Proc. 7th Inter. Congr. Med. Mal., 2: 260.

Tavares 1971. Rev. Assist. Méd. Mines Gerais, 22: 183-190.

Coura, 1974. (cit. Freitas, 1974-1975).

Ibid.

Ibid.

Ibid.

Dubous et al., 1977. Com. XIII Congr. Soc. Bras. Med. Trop. Brasília.

Castro Filho \& Silveira, 1979. Rev. Bras. Malar. D. Trop., 31: 85-98.

Dias, 1982. Rev. Goiana Med., 28:97-102.

Ibid.

Camargo et al., 1984. Rev. Inst. Med. Trop., 26:192-204.

Santos et al., 1969. Rev. Soc. Bras. Med. Trop., 3: 51-52.

Barros et al., 1975. Rev. Inst. Med. Trop. S. Paulo, 17: 319-329.

Castro Filho \& Silveira, 1979. Rev. Bras. Malor. D. Trop., 31: 85-98.

Barros et al., 1980. Rev. Pat. Trop., S. Paulo, $9: 153-156$.

0.32 Camargo et al., 1984. Rev. Inst. Med. Trop., 26: 192-204. 


\begin{tabular}{|c|c|c|c|c|c|}
\hline State & Localities & Year & $\begin{array}{l}\text { Sample } \\
\text { size }\end{array}$ & $\begin{array}{c}\% \\
\text { Positivity }\end{array}$ & References \\
\hline & Rio de Janeiro & 1961 & 435 & 1.8 & $\begin{array}{l}\text { Silva et al., 1961. Arq. Bras. Med., 51: 35- } \\
38 \text {. }\end{array}$ \\
\hline & Rio de Janeiro & 1966 & 4,595 & 1.26 & Coura et al., 1966. Hospital, 69: 991-998. \\
\hline & Rio de Janeiro & 1966 & 319 & 0.0 & Ibid. \\
\hline & Rio de Janeiro & 1966 & 2,242 & 3.56 & lbid. \\
\hline & & 1967 & 25,508 & 0.52 & $\begin{array}{l}\text { Gonzaga et al., 1967. Arq. Bras. Med., 54: } \\
289-301 \text {. }\end{array}$ \\
\hline & Caxias & 1971 & 110 & 0.055 & $\begin{array}{l}\text { Coura et al., 1971. Rev. Soc. Bras. Med. } \\
\text { Trop., 5: 123-129. }\end{array}$ \\
\hline & Caxias & 1975 & 597 & 0.0067 & $\begin{array}{l}\text { Coura \& Pentana, 1975. Rev. Soc. Bras. } \\
\text { Med. Trop., 9: 83-87. }\end{array}$ \\
\hline & & 1979 & 22,275 & 2.5 & $\begin{array}{l}\text { Castro Fitho \& Silveira, 1979. Rev. Bras. } \\
\text { Molar. D. Trop., 31:85-98. }\end{array}$ \\
\hline & & 1984 & & 1.75 & $\begin{array}{l}\text { Camargo et al., } 1984 . \text { Rev. Inst. Med. Trop., } \\
\text { 26:192-204. }\end{array}$ \\
\hline \multirow[t]{23}{*}{ Sāo Paulo } & & 1949 & 500 & 67.4 & $\begin{array}{l}\text { Ramos et al., 1949. Arq. Bras. Cardiol., 2: } \\
\text { 111-162. }\end{array}$ \\
\hline & São Paulo & 1950 & 500 & 1.0 & Faria et al., 1950. Folia Clin. Biol., 16: 158. \\
\hline & Sāo Paulo & 1951 & 92 & 7.2 & Faria, 1951. Folia Clin. Biol, 17: 113-117. \\
\hline & São Paulo & 1952 & 826 & 2.5 & Freitas et al., 1952. Hospital, 41:229-236. \\
\hline & São Paulo & 1952 & 796 & 1.7 & Ibid. \\
\hline & Laranjal Paulista & 1952 & 28 & 10.7 & $\begin{array}{l}\text { Alves \& Alves., 1952. Arq. Hig. Saúde Púb., } \\
\text { 17: 109-115. }\end{array}$ \\
\hline & Ribeirão Preto & 1953 & 19 & 21.1 & $\begin{array}{l}\text { Biancalana et al., 1953. Hospital, 44: 745- } \\
749 .\end{array}$ \\
\hline & Santos & 1953 & 94 & 0.0 & Ibid. \\
\hline & São J. Rio Preto & 1953 & 134 & 14.9 & Ibid. \\
\hline & São Paulo & 1953 & 536 & 4.1 & $\begin{array}{l}\text { Passalacqua et al., 1953. Hospital, } 43: 443- \\
447 .\end{array}$ \\
\hline & São Paulo & 1954 & 786 & 5.4 & $\begin{array}{l}\text { Almeida et al., 1954. Amer. J. Trop. Med. } \\
\text { Hyg., 3: 490-494. }\end{array}$ \\
\hline & São Paulo & 1955 & 178 & 1.7 & $\begin{array}{l}\text { Nussenzweig et al. 1955. Rev. Clin. Fac. } \\
\text { Med. S. Paulo, 10:265-283. }\end{array}$ \\
\hline & São Paulo & 1958 & 627 & 2.0 & $\begin{array}{l}\text { Castro \& Uvo, 1958. Arq. Bras. Cardiol, 11: } \\
114-120 \text {. }\end{array}$ \\
\hline & Ribeirão Preto & 1959 & 3,055 & 14.4 & $\begin{array}{l}\text { Freitas \& Siqueira, 1959. (cit. Baldy et al., } \\
\text { 1978). }\end{array}$ \\
\hline & Ribeirão Preto & 1959 & 6,405 & 10.8 & Ibid. \\
\hline & Ribeirāo Preto & 1959 & 626 & 10.9 & 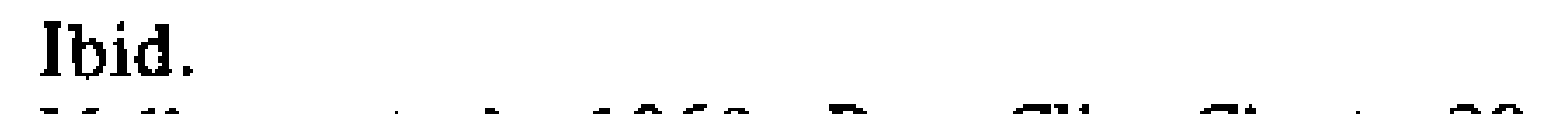 \\
\hline & São Paulo & 1960 & 16,624 & 1.5 & $\begin{array}{l}\text { Mellone et al., 1960. Rev. Clin. Cient., 29: } \\
\text { 101-102. }\end{array}$ \\
\hline & & 1962 & 15,271 & 9.31 & $\begin{array}{l}\text { Coutinho, 1962. Arq. Hig. Saúde Públ., 94: } \\
\text { 317-330. }\end{array}$ \\
\hline & & 1964 & 34,710 & 13.93 & $\begin{array}{l}\text { Silva, 1964. Arq. Hig. Saúde Públ., 29: 129- } \\
140 .\end{array}$ \\
\hline & São Paulo & 1965 & 62,575 & 1.45 & $\begin{array}{l}\text { Mellone \& Pagenotto, 1965. Rev. Hosp. } \\
\text { Clin. Fac. Med. S. Paulo, 20:165-167. }\end{array}$ \\
\hline & Sāo Paulo & 1972 & 15,341 & 1.95 & $\begin{array}{l}\text { Meira et al., 1972. Rev. Medicina, 56: 327- } \\
331 \text {. }\end{array}$ \\
\hline & $\begin{array}{l}\text { Ribeirão Preto } \\
\text { Ribeirão Pre to }\end{array}$ & $\begin{array}{l}1972 \\
1972\end{array}$ & $\begin{array}{l}3,493 \\
4,147\end{array}$ & $\begin{array}{l}9.5 \\
4.3\end{array}$ & $\begin{array}{l}\text { Volpon et al., 1972. (cit. Baldy, 1978). } \\
\text { lbid. }\end{array}$ \\
\hline & Ribeirāo Preto & 1976 & 1,000 & 10.10 & $\begin{array}{l}\text { Vichi et al., 1976. Arq. Bras. Cardiol., 29: } \\
87 .\end{array}$ \\
\hline
\end{tabular}

SOUTH

Paraná

Londrina

1958

Londrina

Londrina

Londrina
1958

1978

1978
Brofman, 1958. Arq. Bras. Cardiol, 11: 209-210.

Queiroz \& Pascual, 1958. Rev. Méd. Paraná, 27: 27-30.

Baldy et al., 1978. Rev. Saúde Públ., Sāo Paulo, 12: 409-416.

Ibid. 


\begin{tabular}{|c|c|c|c|c|c|}
\hline State & Localities & Year & $\begin{array}{c}\text { Sample } \\
\text { size }\end{array}$ & $\begin{array}{c}\% \\
\text { Positivity }\end{array}$ & References \\
\hline & & $\begin{array}{l}1979 \\
1984\end{array}$ & 51,959 & $\begin{array}{l}7.5 \\
4.0\end{array}$ & $\begin{array}{l}\text { Castro Filho \& Silveira, 1979. Rev. Bras. } \\
\text { Malar. D. Trop., 31:85-98. } \\
\text { Camargo et al., 1984. Rev. Inst. Med. Trop., } \\
\text { 26: 192-204. }\end{array}$ \\
\hline Santa Catarina & & $\begin{array}{l}1968 \\
1979 \\
1984\end{array}$ & $\begin{array}{r}1,104 \\
23,833\end{array}$ & $\begin{array}{l}0.09 \\
2.4 \\
1.39\end{array}$ & $\begin{array}{l}\text { Salgado \& Pellegrino, 1968. (cit. Barretto, } \\
\text { 1979). } \\
\text { Castro Filho \& Silveira, 1979. Rev. Bras. } \\
\text { Malar. D. Trop., 31:85-98. } \\
\text { Camargo et al., 1984. Rev. Inst. Med. Trop., } \\
\text { 26: 192-204. }\end{array}$ \\
\hline
\end{tabular}

Rio Grande

do Sul

Encruzilhada Sul
São Jerônimo
Rosário do Sul
Itaqui
Santa Rosa
Herval do Sul,
Lavar do Sul,
Caçapava do Sul

1957

1959

1959

1959

1959

1959

1964

$5,460 \quad 23.9$

$976 \quad 18.9$

$1,822 \quad 18.1$

$1,265 \quad 27.1$

$1,394 \quad 31.9$

$\begin{array}{ll}1,872 & 9.7\end{array}$

$\begin{array}{ll}31,646 & 2.8\end{array}$
1977

$1,041 \quad 18.8$

$5,530 \quad 17.59$

Pelotas

1979

1984
Brant et al., 1957. Rev. Bras. Malar. D. Trop., 9: 141-176.

Sessen \& Annt, 1959. Rev. Med. Rio Grande do Sul, 15: 133-142.

Ibid.

Ibid.

Ibid.

Ibid.

Mello et al., 1964 (cit Coura, 1966. Rev. Bras. Malar. D. Trop., 18:9-98).

Baruffa \& Alcantara, 1973. Rev. Soc. Bras. Med. Trop., 7: 329-332.

Baruffa \& Alcantara, 1977. Com. XIII Congr. Soc. Bras. Med. Trop., Bras.

Baruffa, 1979. Rev. Inst. Med. Trop., S. Paulo, 21: 37-42.

Castro Filho \& Silveira, 1979. Rev. Bras. Malar. D. Trop., 31: 85-98.

8.84 Camargo et al., 1984. Rev. Inst. Med. Trop., 26: 192-204.

\section{MIDWEST}

Mato Grosso

197921,762

1984

\section{Mato Grosso}

do Sul

1984

Goiás

Rio Verde
Goiânia
Trindade
Hidrolândia
Goiânia
Goiânia
Jataí

2.3

Castro Filho \& Silveira, 1979. Rev. Bras. Malar. D. Trop., 31: 85-98.

Camargo et al., 1984. Rev. Inst. Med. Trop.,

2.46 Camargo et al., 1984. Rev. Inst. Med. Trop., 26:192-204.

2.55 Freitas \& Mendonça, 1951. Hospital, 39: 251-261.

Freitas \& Figueiredo, 1951. Arq. Hig. Saúde Públ., 16: 227-230.

Ibid.

Ibid.

$1,474 \quad 11.0 \quad$ Alexandre, 1964 (cit. Rezende, et al., 1965. Rev. Goiana Med. 11: 35-47). Campos et al., 1975. Rev. Soc. Bras. Med. Trop., 9: 165-174.

Camargo et al., 1976. Com. XII Congr. Soc. Bras. Med. Trop., Belém.

Castro Filho \& Silveira, 1979. Rev. Bras. Malar. D. Trop., 31: 85-98.

7.4 Camargo et al., 1984. Rev. Inst. Med. Trop., 26: 192-204. 26: 192-204.
1951 239

1951

1951

1951

1964

1975

1976

1979

1984 


\begin{tabular}{lcccc}
\hline Localities & Year & $\begin{array}{c}\text { Sample } \\
\text { size }\end{array}$ & $\begin{array}{c}\% \\
\text { Positivity }\end{array}$ & References \\
\hline Distrito Federal & 1960 & 242 & 0.413 & $\begin{array}{l}\text { Silva \& Queiroz, 1960. J. Bras. Med., 2: 483- } \\
488 . \\
\text { Tosta et al., 1977. Com. XII Congr. Soc. } \\
\text { Bras. Med. Trop., Brasilia. }\end{array}$ \\
\hline
\end{tabular}

\section{REFERENCES}

CABELlo, P. E. \& KRIEGER, H., 1990. GENIOC, uma programateca de genética (in press).

CAMARGO, M. E.; SILVA, G. R. da; CASTILHO, E. A. \& SILVEIRA, A. C., 1984. Inquérito soroló- gico da prevalência de infecção chagásica, Brasil, 1975-1980. Rev. Med Trop., 26: 192-204.

CASTRO FILHO, J. \& SILVEIRA, A. C., 1979. Distribuição da doença de Chagas no Brasil. Rev. Bras. Malar. D. Chages., Brasflia, 31: 85-98. 